\title{
DWT BASED ENERGY DETECTION SPECTRUM SENSING METHOD FOR COGNITIVE RADIO SYSTEM
}

\author{
Muntaser S. Falih ${ }^{1}$, Hikmat N. Abdullah ${ }^{2}$ \\ ${ }^{1,2}$ College of Information Engineering, Al-Nahrain University, Baghdad, Iraq \\ mntasersaleem@gmail.com ${ }^{1}$, hikmat.abdullah@coie-nahrain.edu.iq ${ }^{2}$ \\ Received:2/02/2020, Accepted:5/05/2020
}

\begin{abstract}
In this paper a new blind energy detection spectrum- sensing method based on Discreet Wavelet Transform (DWT) is proposed. The method utilizes the DWT sub-band to collect the received energy. The proposed method recognizes the Primary User (PU) signal from noise only signal by using the differences in the collected energy in first and last sub-bands of one DWT level. The simulation results show that in the AWGN channel, the proposed method improves detection probability by a factor of $40 \%$ at Signal to Noise Ratio (SNR) equals -13 dB compared to Conventional Energy Detector (CED). Furthermore, the proposed method reduces the number of sensed samples to $50 \%$ on what in the traditional method. Thus, the proposed method achieves shorter sensing time and consumes lower Energy which makes it suitable for Cognitive Radio ( CR) applications where only limited energy like device battery is available.
\end{abstract}

keywords: Cognitive radio, DWT, Energy detection, Spectrum sensing.

\section{INTRODUCTION}

The massive demands on wireless communication equipment lead to a shortage in available spectrum resources. By using Cognitive Radio (CR) technology, spectrum resource can be optimized in usage based on Dynamic Spectrum Allocation (DSA) . Simply, CR is the radio system that can award its operating environment with regards to operation frequency, coding type, polarity and so on. In CR the unlicensed user or Secondary users (SU) can utilize the resources that allocated for the licensed user or Primary Users (PU). The most sensitive phase in CR life cycle is the spectrum sensing. Spectrum sensing (SS) is the process which detect the presence of PU achieved by SU transceiver in order to utilize PU allocated spectrum. This allocated spectrum is called "hole" or "white space" The SS most be enough accurate to avoid PU interference [1],[2]. In literature the basic SS approaches are: Matched Filter (MF), Cyclostationary and Energy Detection (ED). In MF the SS method, the SU receiver detects PU signal based on its specification by designing a filter compatible with the received signal type that gives maximum crosscorrelation. This method assumes that SU know the PU signal specification. In Cyclostationary SS method, the SU detects the PU signal based on finding semi- periodic feature that is related to modulated signal type. In the last approach, i. e. ED, the PU signal is detected based on the received signal power using comparison with pre- defined threshold value. ED is also called blind SS method. It gives fast SS response but suffers from reduced accuracy (i. e. detection probability) in distinction between PU and noise signals at low SNR values [3],[5] In this paper a new ED based on Discreet Wavelet Transform (DWT) is proposed to improve the detection probability of the energy detector.

\section{RELATED WORK}

There are many efforts dealt with developing spectrum sensing of cognitive radio system. These works are divided into two groups. The first group used DWT to achieve spectrum sensing mechanism. For example, in [6] the authors used DWT 
for identification of spectrum holes in wide band frequency via whole edge detection. In [7], the authors proposed novel edge detection method for wide band spectrum sensing using both Continuous Wavelet Transform (CET) and DWT. In [8] , the authors proposed sensing method based on extracting spectrum features using DWT. The second group utilized other features to detect spectrum holes by recognition of the PU signal from the noise signal. For example in [9] the authors introduced a sensing method using statistical parameter which represents the ratio between variance and mean values of energy as an indicator whether the received signal is an PU or noise alone. In [10], the authors proposed a spectrum sensing method based on using noise uncertainty factor to update the threshold value of the energy detector. In [11], the authors proposed an adaptive spectrum sensing method based on higher order moment and compare it with multilevel threshold for decision stage. There are other efforts to develop CED by utilizing another transformation approaches. For example, in [12] the authors designed a new energy based spectrum sensing using Discrete Cosine Transform (DCT) instead of Discrete Fourier Transform (DFT). However, this method does not take into account the noise extraction using DCT coefficient's relationship. In this paper, a new energy detection spectrum sensing method based on DWT is proposed. This method is designed to recognize the PU embedded in noise using the variation of collected energy in first and last sub- bands of one level DWT decomposition. This variation is related to the nature of first sub- band in DWT which contain the most signal energy (information) on the contrary to noise signal that has no information. Thus all sub- band is approximately collecting the same amount of energy. The detection mechanism of this method will be explained in the later sections.

\section{Conventional Energy Detector ( CED)}

Conventional Energy Detector (CED) is the simple context of ED method in which SU accumulates the received energy with respect to a certain number of samples Ns. The binary hypothesis used in the detection criteria is as follows [13] :

$$
y(n)=\left\{\begin{array}{cc}
w(n) & ; H_{0} \\
s(n)+w(n) & ; H_{1}
\end{array}\right.
$$

Where $y(n)$ is the received sample, $w(n)$ is the Additive White Gaussian Noise AWGN sample collected from the transmission channel, $s(n)$ is the PU signal sample, $H_{0}$ and $H_{1}$ are null and power hypothesis respectively. The CED test statistics of received sample is defined as follows:

$$
T=\frac{1}{N_{s}} * \sum_{n=1}^{N s}|y(n)|^{2}
$$

Where $N_{s}$ is the total number of received samples. The distribution of $\mathrm{T}$ is chi- squared with $2 N_{s}$ degrees of freedom [14]. The received samples are compared with predefined threshold computed based on Constant False Alarm probability (CFA) [15]- [17] . The performance of the detection in energy detector schemes is evaluated based on Receiver Operation Characteristics (ROC) that showing the detection probability (PD), False Alarm Probability (PFA), Signal to Noise Ratio (SNR), and threshold $\lambda$. ROC parameters are computed in AWGN as follows [14].

$$
P_{D}=P\left[T>\left.\lambda\right|_{H_{1}}\right] Q\left(\frac{\lambda-N_{s}(1+S N R)}{\sqrt{2 N_{s}(1+S N R)}}\right)
$$

https://ijict.edu.iq 


$$
P_{F A}=P\left[T>\lambda \mid H_{0}\right] Q\left(\frac{\lambda-N_{s}}{\sqrt{2 N_{s}}}\right)
$$

Where $Q(\cdot)$ is complementary Q- function (Marcum), PD represents the correct detection when PU is present, PFA represents false detection alarm that indicates the presence of PU but actually it is not there. PD and PFA in Equation 3 and Equation 4 are calculated in case of unity noise variance. From Equation $4, \lambda$ is calculated as follows:

$$
\lambda=\sqrt{2 N_{s}} Q^{-1}\left(P_{F A}\right)+N_{s}
$$

Where $Q^{-1}$ is inverse Q- function. The system model block diagram of CED depicted in Fig. 1 [13].

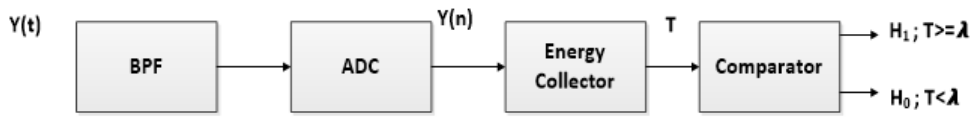

Figure 1: CED method block diagram

\section{The Proposed Method}

The method proposed method utilizes the variation in collected energy at first and last sub- bands of one level decomposition DWT. Fig. 2 shows the block diagram of the proposed method. The detection mechanism in this method can be explained as follows: the received signal is first digitized, and then the signal is DWT transformed using Daubechies wavelet [18]. The energies in first sub-band ELL and last sub-band EHH of the transformed signal as well as the Average Ratio (AR) of them are calculated using Equation 6, Equation 7, and Equation 8 respectively.

$$
\begin{gathered}
E_{L L}=\frac{1}{L L} \sum_{k=1}^{L L}\left|Y L L(k)^{2}\right| \\
E_{H H}=\frac{1}{H H} \sum_{k=1}^{H H}\left|Y H H(k)^{2}\right| \\
A R=\frac{E L L}{E H H}
\end{gathered}
$$

Finally, AR is compared with a certain threshold to decide whether the received signal is PU $\left(H_{1}\right)$ or frequency hole $\left(H_{0}\right)$ . Based on AR the received signal can be recognized either noise signal or PU signal. The reason behind showing this comparison, is to provide the required decision that can be explained as follows: in noise case (absence of PU signal) all DWT sub-bands will collect approximately the same amount of energy, so AR value will be around 1. On the contrary, a case when PU is present, most of the energy will be collected in LL sub- band which gives AR value much greater than 1. Fig. 3 reveals the proposed method full flowchart. The evaluation of AR parameter for both cases when PU is present or absent is achieved at $\mathrm{SNR}=8 \mathrm{~dB}$ and $-8 \mathrm{~dB}$, as shown in Fig. 4 and Fig. 5 respectively. From these figures, it can be seen that AR is approximately unity in case of PU is absent (noise only signal) and is highly above 1 in case of PU is present depending on SNR value. So, a new threshold value slightly greater than 1 can be used to recognize PU from noise signal successfully. 


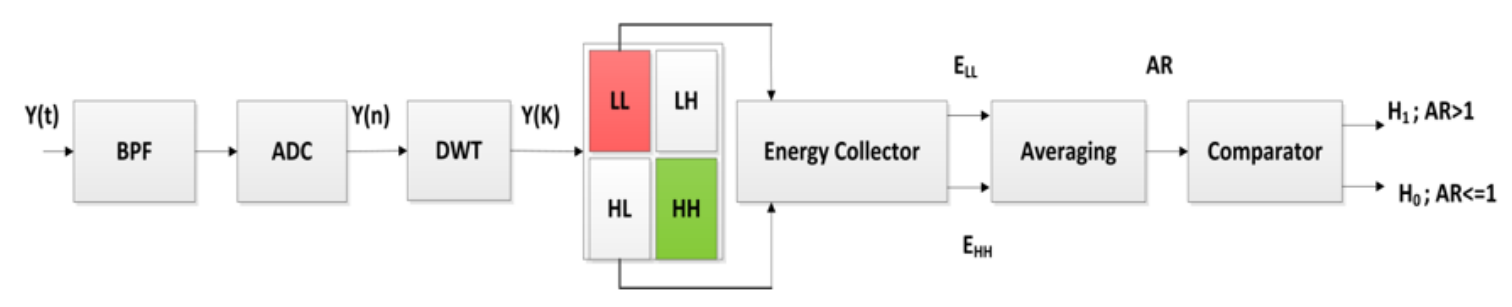

Figure 2: Block diagram of the proposed method

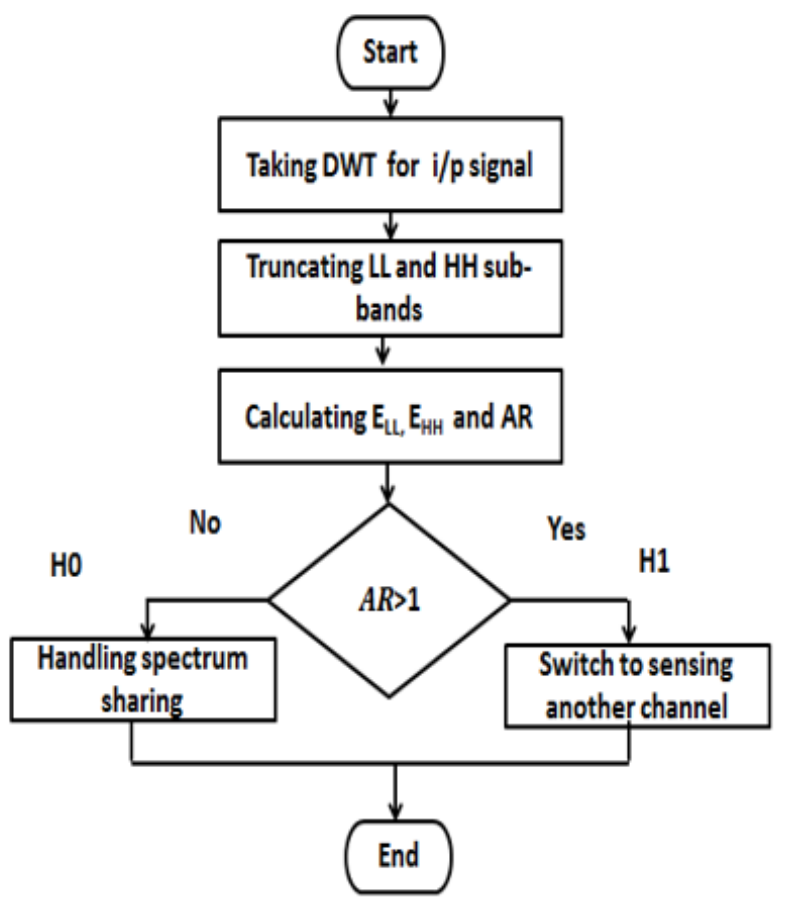

Figure 3: Flowchart of proposed method 


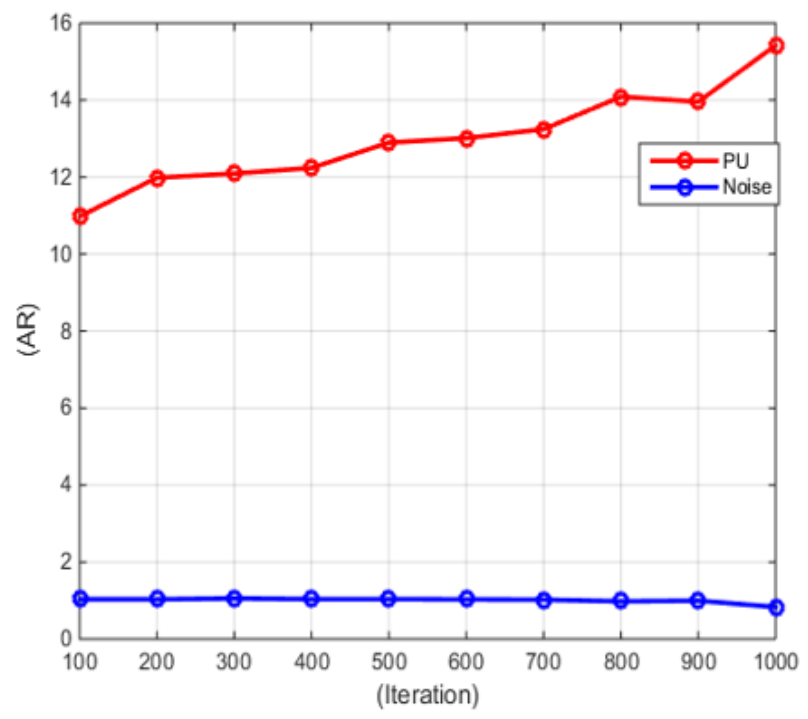

Figure 4: $\mathrm{AR}$ parameter testing at $\mathrm{SNR}=8 \mathrm{~dB}$

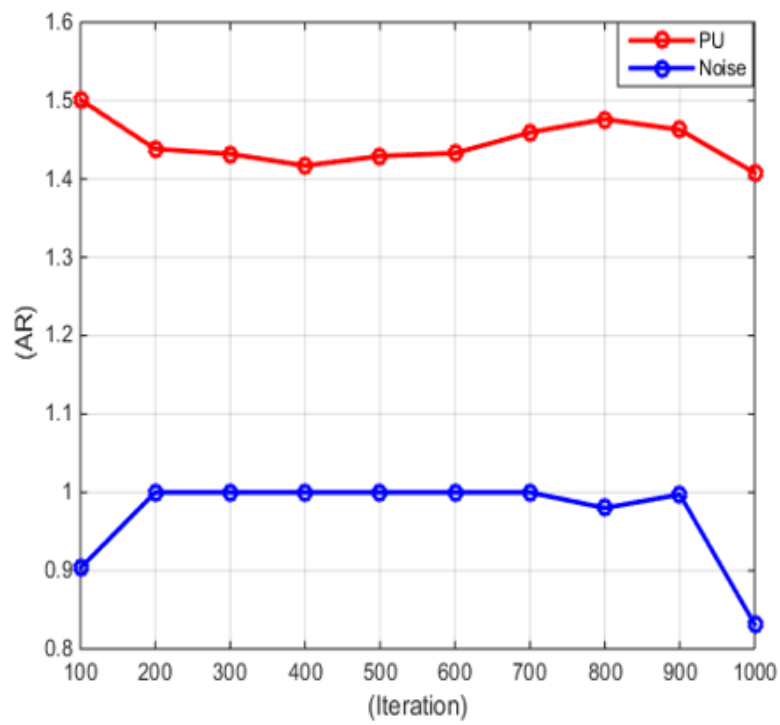

Figure 5: AR parameter testing at $\mathrm{SNR}=-8 \mathrm{~dB}$

\section{RESUlts}

The proposed method is simulated using MALTAB package. The evaluation has been tested for Local Sensing (LS) noncooperative scenario showing the ROC with different values of NS, PFA and channels types. The simulation parameters used are given in Table I. 
TABLE I

SimUlation PARAMETERS

\begin{tabular}{|c|c|}
\hline Parameter & Value \\
\hline PU signal type & QPSK \\
\hline Channel type & AWGN and rayleigh fading \\
\hline$P_{F A}$ & $10^{-} 3$ \\
\hline Number of samples $N_{s}$ & 1500,1000 and 500 \\
\hline SNR range & -20 to $0 \mathrm{~dB}$ \\
\hline
\end{tabular}

Fig. 6 shows the comparison between proposed method and CED in AWGN when $N_{s}=1500$ and PFA=0.001. From this figure it can be seen that the proposed method outperforms CED method in detection probability especially at low SNR. For instance, detection probability increased from 0.5 to 0.9 with improvement factor of $40 \%$ at $\mathrm{SNR}=-10 \mathrm{~dB}$ and from 0.1 to 0.6 with improvement factor of $50 \%$ at $\mathrm{SNR}=-13 \mathrm{~dB}$. The other advantage can be gained in proposed method is that the detection probability reached unity $3 \mathrm{dBs}$ earlier than CED. For instance, in proposed method PD=1 is achieved at $\mathrm{SNR}=-8 \mathrm{~dB}$ while in $\mathrm{CED}$ it is achieved at $\mathrm{SNR}=-5 \mathrm{~dB}$. Fig. 7 shows the evaluation of proposed method against CED in Rayleigh fading channel with NS $=1500$ and $\mathrm{PFA}=0.001$. It can be noticed in this figure it the immunity of the proposed method to fading impact which leads to enhance detection probability compared to CED. For example, at $\mathrm{SNR}=-15 \mathrm{~dB}$ and $-8 \mathrm{~dB}$ the detection probability is enhanced by improvement factor of $35 \%$ and by $20 \%$ respectively. Another test performed is the performance of the proposed method in terms of detection probability versus SNR with $N_{s}$ as a parameter as shown in Fig. 8. The selected numbers of received sample in the simulations are $N_{s}=1500,1000$, and 500. Fig. 8 depicts that proposed method has acceptable flexibility in selecting the number of received samples with somewhat closed performance especially at SNR values greater than $-6 \mathrm{~dB}$. This feature is very interesting for applications that require short sensing time with saving in energy consumption. The other objective of our simulations is to evaluate the performance of proposed system from Energy Consumption (EC) point of view. This evaluation is useful for nominating the SS method that is suited for applications that has restrictions on the allowable amounts of consumed energy, for example sensor nodes in remote area where it is not practical to use battery continually. EC depends on detection probability, sensing energy per sample, transmission energy, and the number of sensed samples. The mathematical formula given in Equation 9 is used to compute EC [19] :

$$
E C=N_{s} * E_{s s}+(1-P D) * E_{t}
$$

Where $N_{s}$ is number of received (sensed) samples, $E_{s s}$ is sensing energy per sample, PD is the detection probability and $E_{t}$ is the transmission energy. Fig. 9 shows comparison of EC between the proposed and CED methods. It can be seen in this figure that the proposed method has an efficient EC performance compared to CED. For instance, EC is improved by $155 \%$ and $135 \%$ at $\mathrm{SNR}=-14 \mathrm{~dB}$ and $-12 \mathrm{~dB}$ respectively. The reason for this EC improvement is that the proposed method uses only $50 \%$ of sensed samples because of using the energy of two- sub bands of the transformed signal out of four, while in CED the entire available spectrum with all sensed samples is used. The performance evaluation of sensing time of the proposed system is also investigated. Sensing time very important parameter in design of spectrum sensing method. It must be short as much as possible to increase the throughput of resources utilization. Sensing time mainly depending 
on $N_{s}$ and the time required to process each sample. Sensing Time (ST) is calculated using Equation 10.

$$
T_{s}=N_{s} * S P T
$$

where $N_{s}$ is number of received samples, and SPT is the time required to process each sample (time of operation) . Fig. 10 shows TS evaluation of proposed and CED methods when $N_{s}=100$ and SST $=1 \mu s$. from this figure there is a noticeable difference in sensing time of proposed method compared with CED method. In the proposed method $T_{s}$ is much shorter than in CED. This is related to a smaller number of $N_{s}$ used proposed method as well as the improvement in detection probability. Finally, Fig. 11 shows the comparison between the proposed method and method presented in [7]. This comparison reveals the detection probability performance versus SNR with the same simulation parameters. Form this figure, the proposed method outperforms the of method in [7]. For example at $\mathrm{SNR}=-12 \mathrm{~dB}$ proposed detection probability increased from 0.2 to 0.9 with improvement factor of $70 \%$. Also, at $\mathrm{SNR}=-10 \mathrm{~dB}$ the detection probability is enhanced by factor of 50\%. Another improvement that can be noticed from this figure is that the proposed method detection probability reaches unity value $6 \mathrm{~dB}$ earlier than CED.

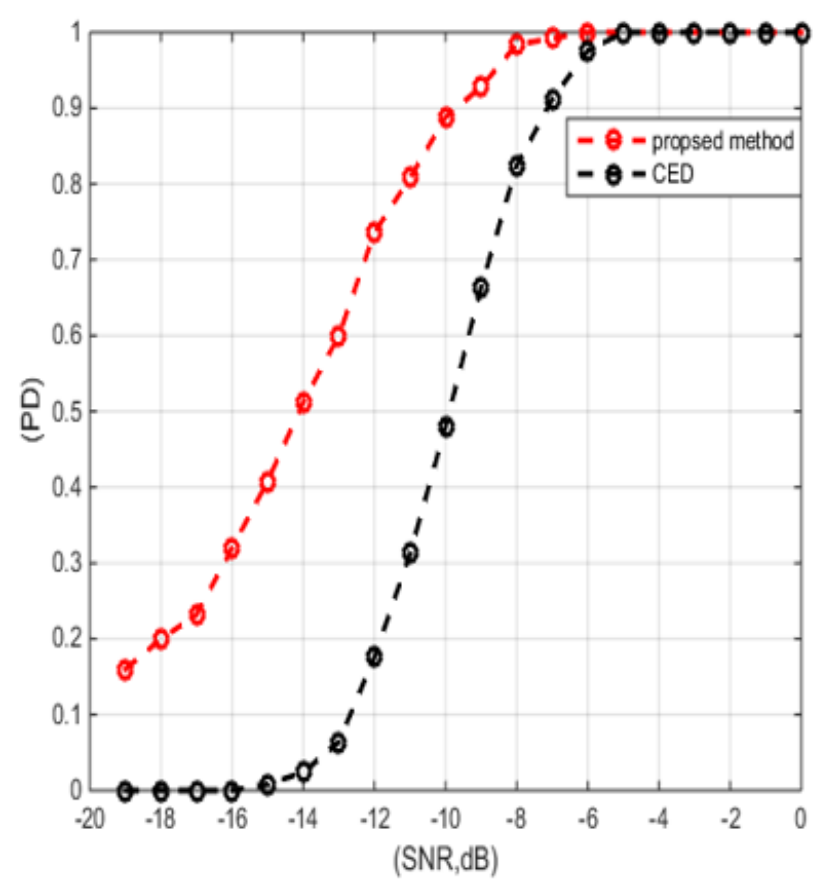

Figure 6: Proposed method performance evaluation in AWGN channel 


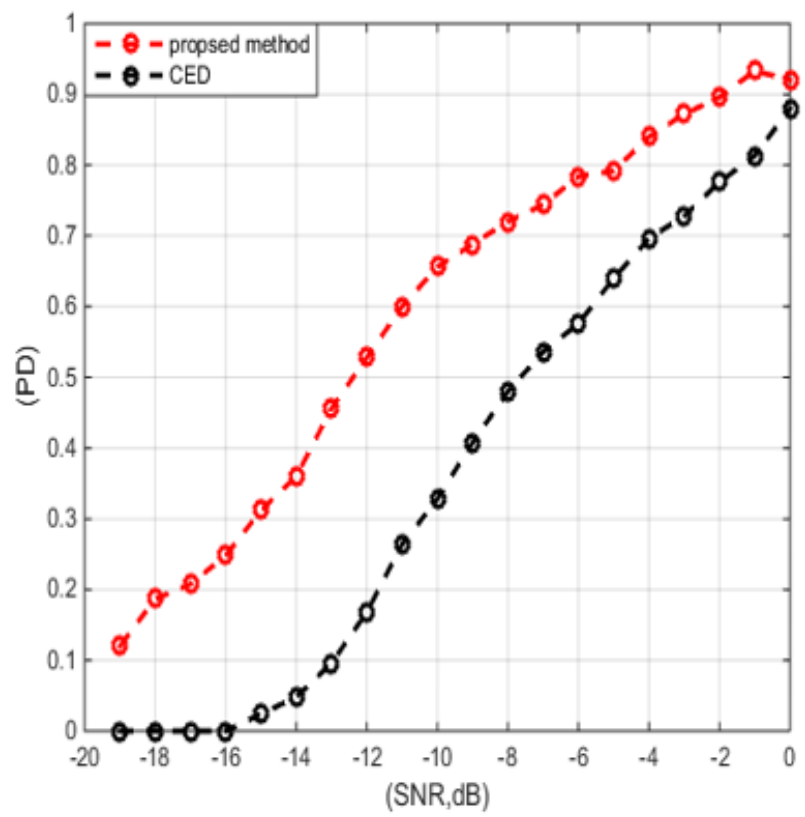

Figure 7: Proposed method performance evaluation in rayleigh fading channel

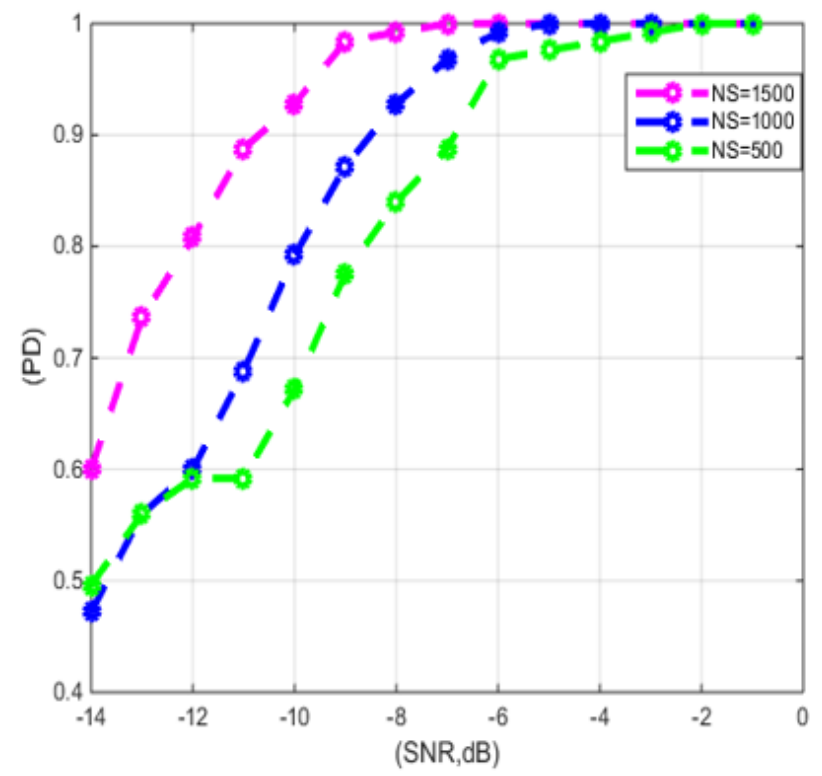

Figure 8: Proposed method performance evaluation in AWGN channel with different samples number 


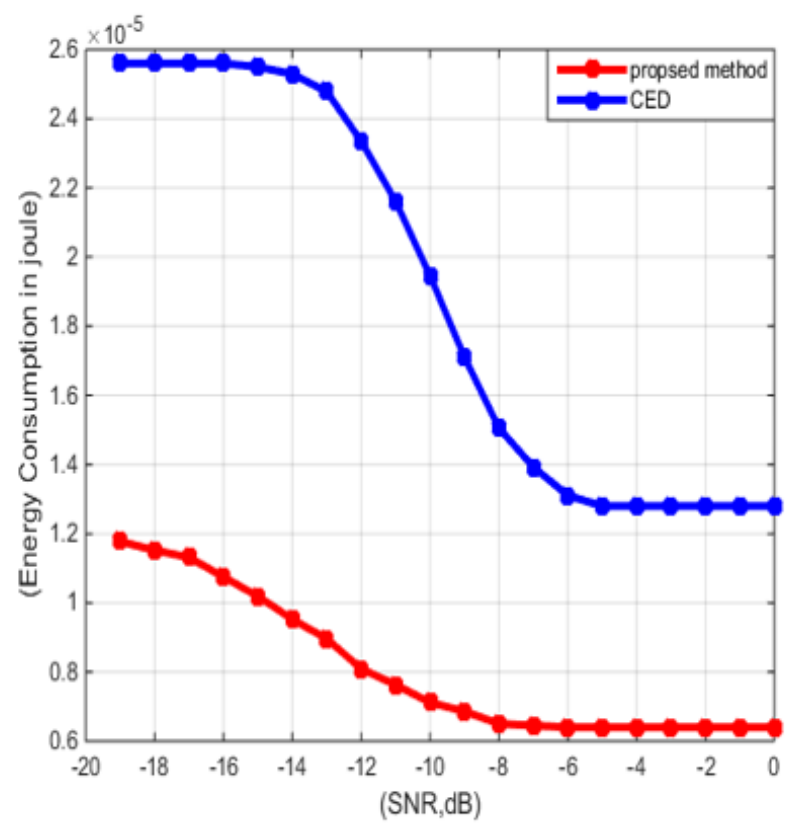

Figure 9: EC Performance of the proposed and CED methods

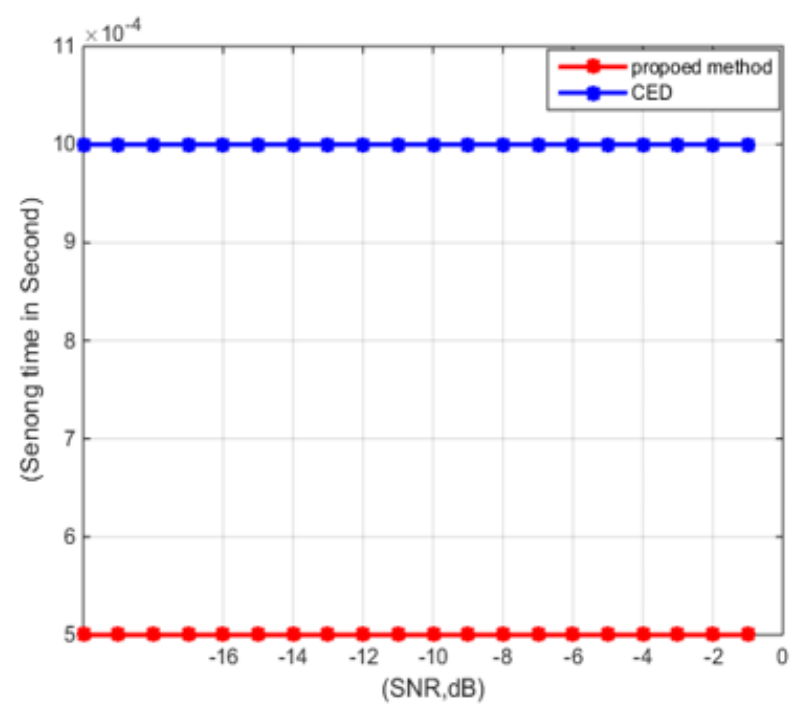

Figure 10: Sensing time performance of the proposed method and CED 


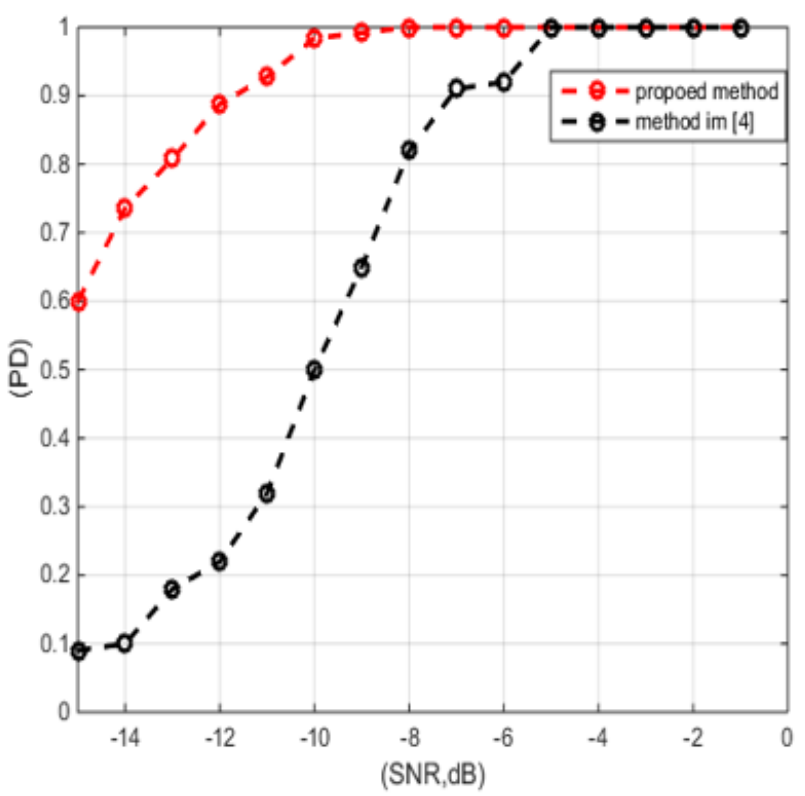

Figure 11: EC performance of the proposed method and method in [7]

\section{Vi. Conclusions}

In this paper, a blind energy detection based on DWT for spectrum sensing in CR proposed. The sensing mechanism of this method is achieved by taking the ratio between the collected energy at first and last sub-bands of one level decomposition DWT. The result shows that the ratio has variation in case of PU presence and absence. So, it has ability to recognize the PU from noise only signal. The justification for this behavior can be seen clearly in PU where the most of energy is collected in a lower sub-band (low frequency or energy) on the contrary for noise signals where all sub-band collect same amount of energy. Moreover, the results show that the proposed method enhances the detection probability especially at low SNR that make it possible for sensing spread spectrum signals. Furthermore, it improves energy consumptions and sensing time which makes it suitable for power limited applications. 


\section{REFERENCES}

[1] M. S. Falih and H. N. Abdullah, "A Combined Spectrum Sensing Method Based DCT for Cognitive Radio System" , Int. J. Electr. Comput. Eng. , vol. 10, no. 2, pp. 1935- 1942, 2020, doi: 10.11591/ ijece. v10i2. pp1935- 1942.

[2] M. I. Khalaf, D. Al- jumeily, and A. L. Eds, "Applied Computing to Support Industry", vol. 2. Springer International Publishing, 2019.

[3] H. Sarvanko, M. Mustonen, A. Hekkala, A. Mammela, M. Matinmikko, and M. Katz, "Cooperative and Noncooperative Spectrum Sensing Techniques Using Welch's Periodogram in Cognitive Radios", 1st Int. Work. Cogn. Radio Adv. Spectr. Manag. CogART 2008, 2008, doi: 10.1109/ COGART.2008.4509987.

[4] X. Chen, H. H. Chen, and W. Meng, "Cooperative Communications for Cognitive Radio Networks- from Theory to Applications" , IEEE Commun. Surv. Tutorials, vol. 16, no. 3, pp. 1180- 1192, 2014, doi: 10. 1109/ SURV.2014. 021414. 00066.

[5] M. S. Falih and H. N. Abdullah, "Evaluation of Different Energy Based Spectrum Sensing Approaches in Cognitive Radio System: Practical Perspective" , vol. 6, no. 12, pp. 2015- 2018, 2018.

[6] A. Kumar, S. Saha, and R. Bhattacharya, "Wavelet Transform Based Novel Edge Detection Algorithms for Wideband Spectrum Sensing in CRNs" , AEU - Int. J. Electron. Commun. , vol. 84, no. November 2017, pp. 100- 110, 2018, doi: 10.1016/ j. aeue. 2017. 11. 024.

[7] S. V. R. K. Rao and G. Singh, "Wavelet Based Spectrum Sensing Techniques in Cognitive Radio" , Procedia Eng. , vol. 38, pp. 880- 888, 2012, doi: 10. $1016 /$ j. proeng. 2012. 06. 111.

[8] Karthik Divakaran , Naveen Manikandan and Shri Hari, "Wavelet Based Spectrum Sensing Techniques for Cognitive Radio- A Survey" , International Journal of Computer Science \& Information Technology (IJCSIT), Vol. 3, No. 2, April 2011.

[9] W. Zhang, X. Jing, and J. Li, "An Energy Detection Based on Coefficient of Variation for Spectrum Sensing in Cognitive Radio" , Lect. Notes Electr. Eng., vol. 473, pp. 87- 94, 2018, doi: 10.1007/ 978- 981- 10- 7521- 6- 11.

[10] H. M. Farag and E. M. Mohamed, "Improved Cognitive Radio Energy Detection Algorithm Based Upon Noise Uncertainty Estimation" , Natl. Radio Sci. Conf. NRSC, Proc., no. April, pp. 107- 115, 2014, doi: 10.1109/ NRSC. 2014. 6835067.

[11] H. T. Ziboon and A. A. Thabit, "A New Proposed Adaptive Cognitive Radio detection system Based on MLP Neural Network for Different Modulation Schemes", ARPN J. Eng. Appl. Sci., vol. 12, no. 2, pp. 521- 527, 2017.

[12] E. H. Salman et al. , "On the Energy Detection Performance Based Welch's DCT Algorithm in Cognitive Radio Systems" , 1st Int. Sci. Conf. Eng. Sci. , 3rd Sci. Conf. Eng. Sci. ISCES 2018 - Proc., vol. 2018, Janua, pp. 135- 139, 2018, doi: 10.1109/ ISCES. 2018.8340542.

[13] M. S. Falih and N. Abdullah, "Double Threshold with Knowledge Based Decision Spectrum Sensing Method" , vol. 10, no. 1, pp. 2169- 2175, 2018.

[14] J. Song, Z. Feng, P. Zhang, and Z. Liu, "Spectrum Sensing in Cognitive Radios Based on Enhanced Energy Detector" , IET Commun. , vol. 6, no. 8, pp. 805- 809, 2012, doi: 10.1049/ iet- com. 2010. 0536.

[15] H. M. Farag and M. Ehab, "An Efficient Dynamic Thresholds Energy Detection Technique for Cognitive Radio Spectrum Sensing" , 2014 10th Int. Comput. Eng. Conf. Today Inf. Soc. What's Next? , ICENCO 2014, no. April 2015, pp. 139- 144, 2015, doi: 10.1109/ ICENCO. 2014. 7050446.

[16] S. M. Kay, "Steven M. Kay- Fundamentals of Statistical Signal Processing, Volume II- Detection Theory- Prentice Hall ( 1998). pdf." 1968, doi: 10. 1088/ 0004- 637X/ 704/ 2/ 1616.

[17] W. Baldygo, R. Brown, M. Wicks, P. Antonik, G. Capraro, and L. Hennington, "Artificial Intelligence Applications to Constant False Alarm Rate (CFAR) Processing" , 1993 IEEE Natl. Radar Conf. , pp. 275- 280, 1993, doi: 10.1109/ nrc. 1993. 270451.

[18] "Ten Lectures of Wavelets"

[19] H. N. Abdullah and H. S. Abed, "Improvement of Energy Consumption in Spectrum Sensing Cognitive Radio Networks Using an Efficient Two Stage Sensing Method", Acta Polytech., vol. 57, no. 4, pp. 235- 244, 2017, doi: 10. 14311/ AP. 2017. 57. 0235. 